\title{
A MONTE CARLO METHOD FOR SOLVING HEAT CONDUCTION PROBLEMS WITH COMPLICATED GEOMETRY
}

\author{
JUN SHENTU, SUNGHWAN YUN, and NAM ZIN CHO* \\ Korea Advanced Institute of Science and Technology \\ 373-1 Guseong-dong, Yuseong-gu, Daejeon, 305-701, Korea \\ 'Corresponding author. E-mail : nzcho@kaist.ac.kr
}

Received December 28, 2006

Accepted for Publication February 22, 2007

A new Monte Carlo method for solving heat conduction problems is developed in this study. Differing from other Monte Carlo methods, it is a transport approximation to the heat diffusion process. The method is meshless and thus can treat problems with complicated geometry easily. To minimize the boundary effect, a scaling factor is introduced and its effect is analyzed. A set of problems, particularly the heat transfer in the fuel sphere of PBMR, is calculated by this method and the solutions are compared with those of an analytical approach.

KEYWORDS : Monte Carlo, Transport Effect, Heat Conduction, MCNP, Scaling Factor, Boundary Correction, PBMR

\section{INTRODU:TION}

Heat conduction is usually modeled as a diffusion process with the governing differential equation identical in form to that of a neutron diffusion equation under no absorption, no fission and one speed condition $[1,2]$. While neutron diffi.sion approximates the neutron transport phenomena, inversely it is applicable to solve diffusion problems by transport methods [2]. Based on this idea, a new Monte Carlo method for solving heat conduction problems is developed in this paper, which employs the MCNP code, as MCNP with versatile geometrical capabilities is widely used as a Monte Carlo particle transport code [3].

In the development of this method, a scaling factor is introduced to make the transport approximation more diffusive. In acdition, analogous to the extrapolation distance concept in reactor physics [2], a boundary correction is introduced to improve upon the imaccurate results of the transport approximation of the diffusion process near the boundary.

Numerical heat transfer methods such as the finite difference or finite element are well developed [4, 5], and Monte Carlo techniques for heat conduction have also been reported [6-9]. Most of the earlier Monte Carlo methods for heat conduction are based on discretized mesh systems, thus they are inherently limited in the geometry treatment. As in this paper, Fraley et al. uses a "meshless" system but their study does not give proper treatment to boundary conditions [9]. The method in this paper is a "transport theory approximation" of the heat conduction equation with a methodical boundary correction. The heat diffusion is simulated by the particle transport process, and the temperature or heat flux is given by the statistical values or tallies in the Monte Carlo method. Thus, this approach appears limited to the calculation of temperatures at specific points rather than at the entire temperature field. Shortcomings include long computing time and variance due to the statistical results. However, it can treat problems with very complicated geometries such as the heat conduction in a fuel sphere of a Pebble Bed Modular Reactor (PBMR) [10].

The heat transfer within a pebble of PBMR is fundamental to the thermal analysis of the pebble bed reactor. The homogenized or effective heat conductivity of the fuel region could be derived from the volumetric average, harmonic average, or a combination of these values [11]. The calculation results of the transport approximation method in this paper indicate that the effective heat conductivity should be between the volumetric average and the harmonic average.

\section{TRANSPORT THEORY APPROXIMATION TO THE HEAT CONDUCTION EQUATION}

The steady state differential equation of heat conduction 
fo: a stationary, isotropic solid is given by [1]

$$
\nabla \cdot K(\vec{r}) \nabla T(\vec{r})+q^{\prime \prime \prime}(\vec{r})=0
$$

where $K(\vec{r})$ is the thermal conductivity and $q^{\prime \prime \prime}(\vec{r})$ is the internal heat source.

The first type of boundary condition is the prescribed surface temperature:

$$
T\left(\overrightarrow{r_{s}}\right)=f\left(\overrightarrow{r_{s}}\right)
$$

where $\vec{r}_{s}$ is on a boundary surface.

If the surface temperature is uniform, the boundary condition (2) can be transformed to the homogeneous boundary condition, i.e., zero surface temperature, by setting $T=T(\vec{r})-f\left(\vec{r}_{s}\right)$ and substituting into Eq. (1), which doies not change the form of the differential equation.

Therefore, the problem to work with is the heat conduction Eq. (1) and the zero surface-temperature boundary condition.

On the other hand, the steady state, one-speed neutron diffusion equation under isotropic scattering, no absorption, and no fission condition is given by [2]

$$
\nabla \cdot \frac{1}{3 \sum_{s}} \nabla \phi(\vec{r})+S(\vec{r})=0
$$

where $\phi$ represents the neutron flux, $\Sigma_{s}$ is the scattering cross section, and $S$ is the internal neutron source.

Comparing Eq. (1) and Eq. (3), these two equations are formally identical. Hence, solving the heat conduction problem with thermal conductivity $K(\vec{r})$ and source $q^{\prime \prime \prime}(r)$ is equivalent to solving the neutron diffusion problem

with isotropic scattering cross section $\sum_{s}=\frac{1}{3 K(\vec{r})}$ and the in ternal neutron source $S=q^{\prime \prime \prime}$. The corresponding boundary condition is the zero neutron flux on the boundary surface.

The neutron diffusion theory approximates the neutron transport phenomena; the approximation is valid except near the boundary [2]. Thus, inversely it is applicable to simulate the diffusion by transport theory in the interior region. As to the boundary region, a suitable correction is needed, as the transport theory is "approximate" to the diffusion theory near the boundary'.

\footnotetext{
'Here diffusion refers to both the neutron diffusion and the heat diffusion, as they are formally identical except for the absorption term.
}

\section{THE TRANSPORT AND DIFFUSION BOUNDARY CONDITIONS}

The zero flux/temperature boundary in the diffusion does not have an equivalent boundary condition in transport theory. In a general-purpose, generalizedgeometry, coupled neutron/photon/electron Monte Carlo transport code such as MCNP [3], the only way to end a particle history is when the particle leaks out of the system through a vacuum boundary (no absorption).

The vacuum boundary condition can be approximated in the neutron diffusion theory by the "zero incoming partial current":

$$
J_{-}\left(\overrightarrow{r_{s}}\right)=\frac{1}{4} \phi\left(\overrightarrow{r_{s}}\right)+\frac{D}{2} \frac{\partial \phi\left(\overrightarrow{r_{s}}\right)}{\partial n}=0
$$

Analogously, in heat diffusion (with no incoming heat flux), the corresponding vacuum boundary condition can be written as:

$$
\frac{1}{4} T\left(\overrightarrow{r_{s}}\right)+\frac{K}{2} \frac{\partial T\left(\overrightarrow{r_{s}}\right)}{\partial n}=0 .
$$

It is claimed that the solutions of Eq. (1) with different boundary conditions, i.e., the zero surfacetemperature and boundary condition (5), differ only by a constant.

For a one-dimensional homogeneous problem with a symmetrical temperature distribution, the solution of Eq. (1) is:

$$
T(x)=-\frac{q^{\prime \prime \prime}}{2 K} x^{2}+C
$$

where $C$ is a constant assigned to satisfy the boundary conditions. Thus, the solutions differ only by a constant.

For general cases, according to the theory of differential equations, the solution of Eq. (1) is composed by the homogeneous solution and any particular solution [12]:

$$
T(\vec{r})=T_{h}(\vec{r})+T_{p}(\vec{r}) .
$$

The same particular solution can be chosen while the homogeneous solution is allowed to vary in order to satisfy the boundary condition, as the homogeneous solution contains undetermined coefficients. Nevertheless, the homogeneous equation of Eq. (1), which gives the homogenous solution, is precisely the Laplacian equation [13]:

$$
\nabla \cdot K(\vec{r}) \nabla T_{h}(\vec{r})=0
$$

The solution of the Laplacian equation with uniform 
boundary conditions is a constant that equals the boundary value (If the boundary condition $T_{h}\left(\vec{r}_{s}\right)$ is not uniform, it is necessary to consider its "extension" in the whole problem field). Therefore, the solutions with different (but uniform) boundary conditions differ only by a constant over the problem field.

Thus far, the logic is:

First, use a Monte Carlo code such as MCNP to obtain the solution in the interior region of the heat diffusion problem with vacuum boundary condition (5).

Second, subtract the MCNP results by a constant to obtain the desired solution of the heat diffusion problem with zero surface temperature. This procedure shall be termed "solution translation".

However, at this point questions concerning what the subtracted constant is and how the solution near the boundary is obtained arise. These issues are addressed in the subsequent section.

\section{SCALING EFFECT AND BOUNDARY CORREC'ION}

One natural candidate of that constant is the boundary value of the MCNP results that are obtained using the vacuum boundary condition. However, as stated earlier, the transport approximation is not accurate for diffusion theory near the boundary. For this reason, the boundary value of the MCNP results is unreliable and does not equal that constant.

Two approaches can be applied to overcome this problem:

(1) Enlarging the valid range of the transport approximation.

Given that the transport approximation is not valid only near the boundary, if the invalid range is reduced, data that is more reliable near the boundary can be obtained. In the extreme, if the invalid range is reduced until it is close to (or equal to) zero, then all MCNP results, interior or boundary, would be accurate, and the boundary value of MCNP results could be used directly as the constant.

(2) Making a boundary correction. Now that the transport approximation is not valid only near the boundary, if a correction is made near the boundary, then we would obtain the corrected results near the boundary as well as the corrected boundary value to be used as the constant.

These two approaches are illustrated in Fig. 1.

Both approaches are used in the proposed method. In the first approach, the method to enlarge the valid range is to reduce the mean free path in the transport calculation. In reactor physics, it is known that diffusion theory "yields the proper flux only in the interior of the reactor, that is, several mean-free paths away from the reactor boundary [2]." In other words, the invalid range of the approximation is several mean-free paths. Therefore, by

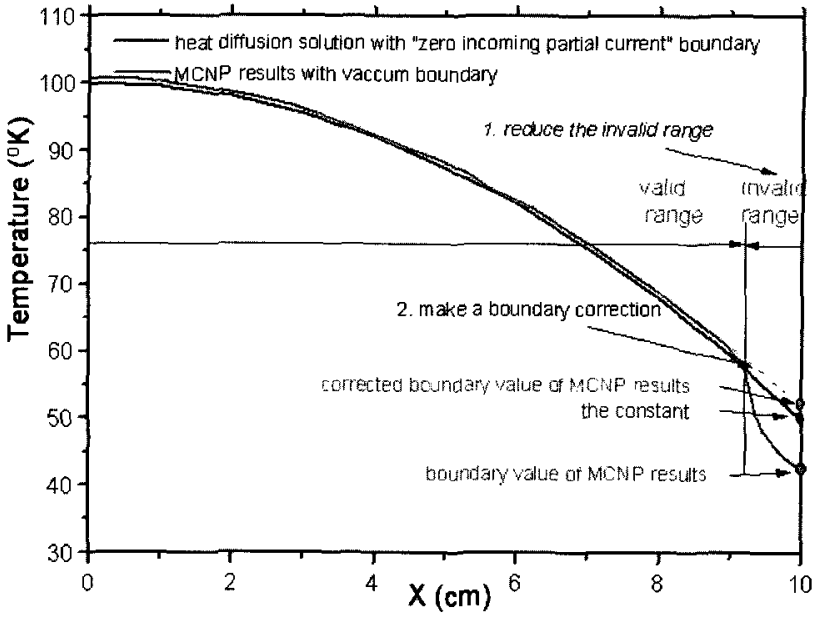

Fig. 1. Illustration of the Valid range, Invalid Range, the Constant, and the Two Procedures Described in the Text

reducing the mean-free path of the problem, the invalid range can be reduced.

The heat conduction Eq. (1) can be rewritten as

$$
\nabla \cdot\left[\frac{1}{\beta} K(\vec{r})\right] \nabla T(\vec{r})+\frac{1}{\beta} q^{\prime \prime \prime}(\vec{r})=0
$$

Although both the thermal conductivity and the internal heat source are scaled by $\beta$, the solutions are identical. $\beta$ is termed "scaling factor". Additionally, the relationship between the mean-free path and $\beta$ is:

$$
\lambda=\frac{1}{\sum_{s}}=\frac{1}{1 /\left(3{ }_{\beta}^{1} K(\vec{r})\right)}=\frac{3 K(\vec{r})}{\beta} .
$$

Therefore, a large $\beta$ gives a small mean-free path, and the small mean-free path gives a transport approximation range that is more valid. This is called "scaling effect".

In another view, if the transport process becomes more diffusive, the approximation is then more accurate. A small mean-free path merely increases the diffusivity of the transport process. However, reducing the mean-free path will unfortunately lead to long computing time: Therefore, "optimal" scaling is a compromise of the approximation accuracy and the computing time.

In the second approach, a boundary correction must be made. The easiest way to do this is to choose points in the interior and make a linear extrapolation to the boundary, as illustrated in Fig. 1. The error induced by the boundary correction depends on two aspects:

(1) How long the correction needs to cover. Here, shorter is better. 
(2) The real temperature distribution near the boundary. If it is linear, then a linear extrapolation would induce a relatively small error.

These can be improved greatly by scaling, as a small mean-free path indicates a short invalid range of transport approximation, and this short invalid range signifies a short boundary distance that needs to be corrected. Moreover, for short distances the real temperature distribution should show more linearity regardless of its real distribution. This explains why the boundary correction is always used in conjunction with scaling.

For the choice of a proper scaling factor and boundary correction distance, the following problems are tested:

Problem 1: a one-dimensional homogeneous slab, thickness $L=10 \mathrm{~cm}, K=0.5 \mathrm{~W} /\left(\mathrm{cm} \cdot{ }^{\circ} \mathrm{K}\right), q^{\prime \prime \prime}=10 \mathrm{~W} / \mathrm{cm}^{3}$ throughout the slab. One side is reflective; the other side is kept at a temperature of zero.

Problem 2: a one-dimensional heterogeneous slab, thickness $L=10 \mathrm{~cm}, q^{m=}=10 \mathrm{~W} / \mathrm{cm}^{3}$ throughout the slab. One side is reflective; the other side kept at a temperature of zero. The heterogeneity comes from the heat conductivities, as follows:

$$
K= \begin{cases}0.2 W /\left(\mathrm{cm} \cdot{ }^{\circ} K\right), & 0 \mathrm{~cm} \leq x \leq 2 \mathrm{~cm}, \\ 0.3 W /\left(\mathrm{cm} \cdot{ }^{\circ} \mathrm{K}\right), & 2 \mathrm{~cm} \leq x \leq 4 \mathrm{~cm}, \\ 0.4 W /(\mathrm{cm} \cdot K), & 4 \mathrm{~cm} \leq x \leq 6 \mathrm{~cm}, \\ 0.5 W /\left(\mathrm{cm} \cdot{ }^{\circ} \mathrm{K}\right), & 6 \mathrm{~cm} \leq x \leq 10 \mathrm{~cm},\end{cases}
$$

The Root Mean Squared Error (RMSE) between the analytical solution and the transport solution (scaled, boundary corrected and translated) are shown as a function of the scaling and boundary correction distance in Figs. 2 and 3 .

In Figs. 2 and 3, each scaling is represented by the dimensionless problem size, i.e. $L / \lambda$ A large dimensionless size denotes a small mean-free path and large scaling factor $\beta$. The zero-boundary-correction distance indicates that no boundary correction is made. Therefore, in this case the boundary value of the MCNP results is directly used as the constant in the solution translation procedure.

In Figs. 2 and 3, if the dimensionless problem size is scaled by less than 10 , the error is comparably large. If the dimensionless problem size is scaled by more than 20 , the error becomes small. With a problem size larger than 20 , the error can be further reduced only slightly while the computing time increases rapidly.

For each scaling, the minimum error occurs when the bcundary-correction distance is approximately 1 mean-free path. However, when the scaling factor is very large, each boundary correction distance results in nearly the same small error, as in this situation the mean free path is very small. Thus, for problems in which it is difficult to determine a boundary correction distance, it is a good practice to

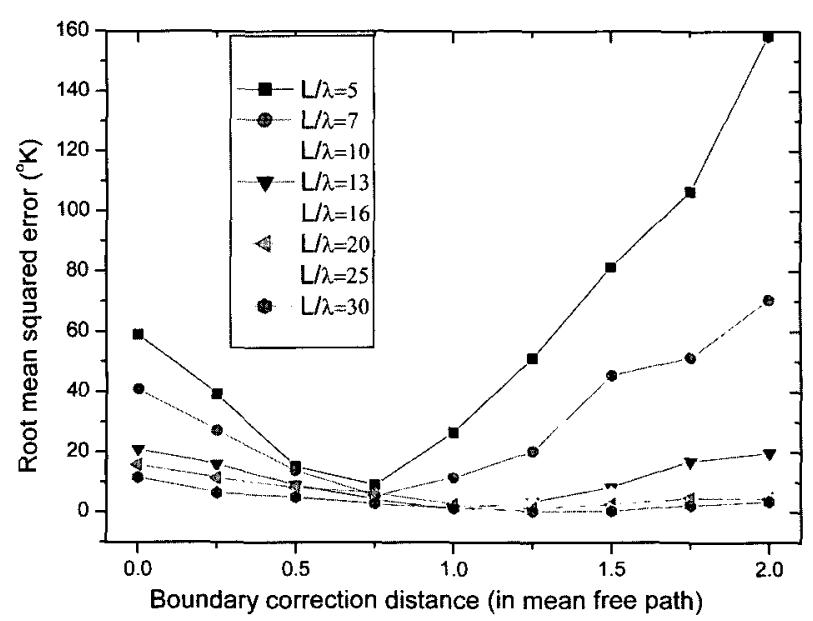

Fig. 2. RMSE of Each Scaling and Boundary Correction Distance for the Homogeneous Problem

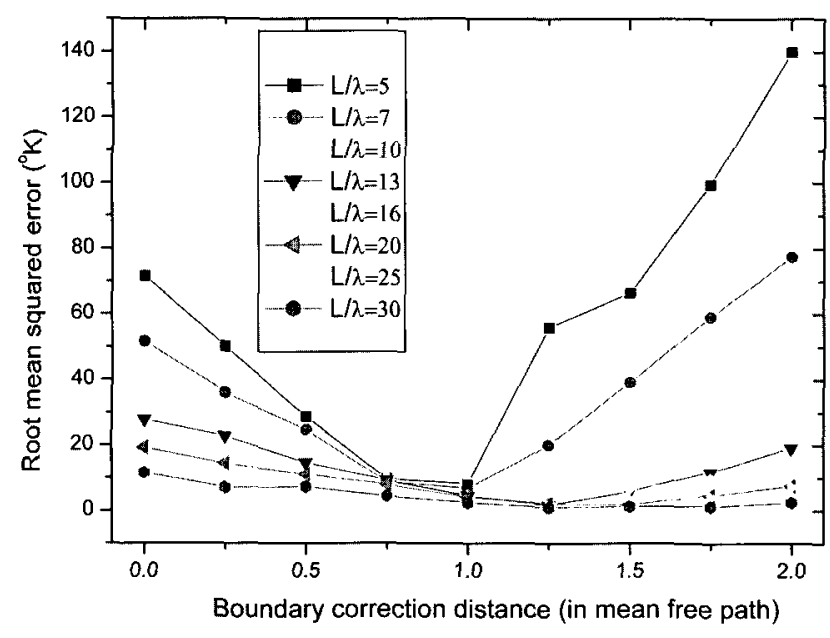

Fig. 3. RMSE of Each Scaling and Boundary Correction Distance for the Heterogeneous Problem

choose a large scaling factor $(\geq 20)$ and skip the boundary correction.

From Figs. 2 and 3, it is clear that the problem size in terms of mean free path should be set between 10 and 20 and that the boundary correction should start from 1 mean -free path away from the boundary. Thus, the scaling factor is:

$$
\beta=\frac{K_{0}}{K_{1}}=\frac{K_{0}}{\lambda_{1} / 3}=\frac{3 \alpha K_{0}}{L},
$$

and the boundary correction distance is:

$$
d=\lambda_{1}=\frac{L}{\alpha},
$$


where $K_{0}$ is the original average heat conductivity, $K_{1}$ is the scaled average heat conductivity (for a heterogeneous problem, the average heat conductivity is used), $\lambda_{1}$ is the scaled mear-free path, and $L$ is the problem size (side length for a rectangle, radius for a sphere, etc.), Here, $\alpha$ represents the dimensionless problem size and is a number between 10 and 20 . It is suggested that for simple geometry, $\alpha$ be set to 10 and for complex geometry, it be set to 20 .

After having obtained the scaling factor, the problem is scaled such that it can be calculated by MCNP:

$$
\begin{gathered}
\sum_{i}=\frac{\beta}{3 K_{i}}, \\
S_{\beta}=q^{\prime \prime \prime} / \beta,
\end{gathered}
$$

where $K_{i}$ is the original heat conductivity of each medium and $\Sigma_{i}$ is the scaled cross section of that medium. $S_{\beta}$ is the scaled source.

As a summary, the procedures to solve the diffusion problem usir.g transport theory are as follows:

Step 1: Choose a scaling factor by (11) and scale up/down the diffusion problem by (13), (14).

Step 2: Solve the scaled problem by a transport code; in this case, this is MCNP.

Step 3: Make a linear correction near the boundary according to (12).

Step 4: Translate the corrected results to satisfy the boundary condition.

These procedures are illustrated in Fig. 4.

\section{CONVECTIIVE BOUNDARY CONDITION}

Thus far, the fixed-temperature boundary condition has been considered, but in many cases, it is necessary to deal with the convective boundary condition. The boundary surface under consideration transfers heat by convection according to Newton's law of cooling to a surrounding temperature:

$$
K_{1} \frac{\partial T\left(\overrightarrow{r_{s}}\right)}{\partial n}=h\left(T_{\infty}-T\left(\overrightarrow{r_{s}}\right)\right),
$$

where $r_{s}$ is on a boundary surface, $\quad \partial T / \partial n$ represents a derivative along the outward normal to the surface, $K_{1}$ is the thermal conductivity of medium $1, h$ is the convective heat transfer coefficient of the convective medium, and $T_{\infty}$ is the bulk temperature of the convective medium.

The detail of the convection mechanism is fairly complicated and is represented by the integral coefficient $h$. Under certain assumptions, the convective boundary condition can be transformed into the fixed temperature boundary cordition.

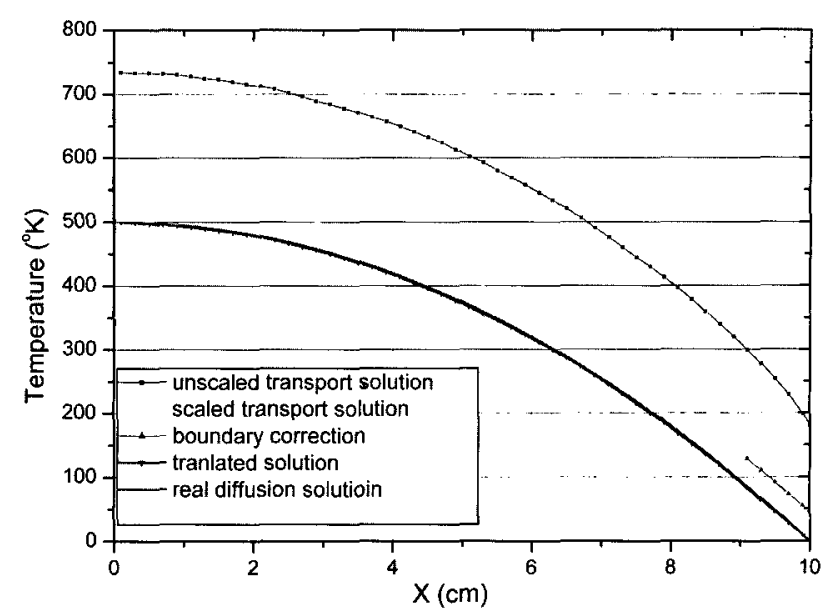

Fig. 4. Method for Solving Diffusion Problems Using Transport Theory

In the following, it is assumed that $T_{\infty}$ is the temperature at a distance $\Delta n$ away from the surface into the convective medium and that the temperature distribution of $\Delta n$ thickness can be approximated as linear:

$$
\frac{\partial T}{\partial n} \simeq \frac{T_{\infty}-T\left(\overrightarrow{r_{s}}\right)}{\Delta n}
$$

This holds everywhere in the added layer with thickness $\Delta n$.

Eq. (15) can then be rewritten as

$$
K_{1} \frac{\partial T\left(\overrightarrow{r_{s}}\right)}{\partial n}=h \Delta n \frac{T_{\infty}-T\left(\overrightarrow{r_{s}}\right)}{\Delta n} \simeq h \Delta n \frac{\partial T(\vec{r})}{\partial n} .
$$

Thus, this layer can be regarded as conduction medium 2 , with the heat conductivity given by $K_{2}=h \Delta n$ [9]. Note that, in spherical geometry, $K_{2}=h \Delta n\left(r_{\mathrm{s}} / r_{\infty}\right)$. Eq. (17) is no more than a continuity expression of heat flux on the interface. Therefore, the convective boundary condition is transformed to the fixed temperature $\left(T_{\infty}\right)$ boundary condition while the boundary surface is extended $\Delta n$ distance.

In the above transformation, in order to guarantee the linearity of the temperature distribution, the thickness $\Delta n$ should be as small as possible. Therefore, using a large scattering cross section $\boldsymbol{\Sigma}_{\mathrm{s} 2}$ for medium 2 (that renders $\Delta n$ small), the linearity of the temperature distribution in medium 2 can be assured.

However, in terms of transport theory, the thickness of the added layer in the unit of the mean-free path is:

$$
\frac{\Delta n}{\lambda}=\frac{\Delta n}{1 / \sum_{s}}=\frac{\Delta n}{3 K}=\frac{\Delta n}{3 h \Delta n}=\frac{1}{3 h} .
$$


Thus, the dimensionless thickness does not depend on the choice of $\Delta n$; in other words, the thickness is always a constant that is $\frac{1}{3 h}$ times its mean-free path. For this reason, the choice of $\Delta n$ should be insensitive.

To verify the above statement, the following problem was tested:

Problem 3: a one-dimensional homogeneous slab, thickness $L=10 \mathrm{~cm}, K=0.5 \mathrm{~W} /\left(\mathrm{cm} \cdot{ }^{\circ} \mathrm{K}\right), q^{\prime \prime \prime}=10 \mathrm{~W} / \mathrm{cm}^{3}$ throughout the slab. One side is reflective; the other side has the convective boundary condition of $h=0.1 \mathrm{~W} / \mathrm{cm}^{2} \cdot{ }^{\circ} \mathrm{K}, T_{\infty}=0$. The transport solutions (deep scaled and translated) with different $\Delta n$ values as well as the analytical solution are plotted in Fig. 5.

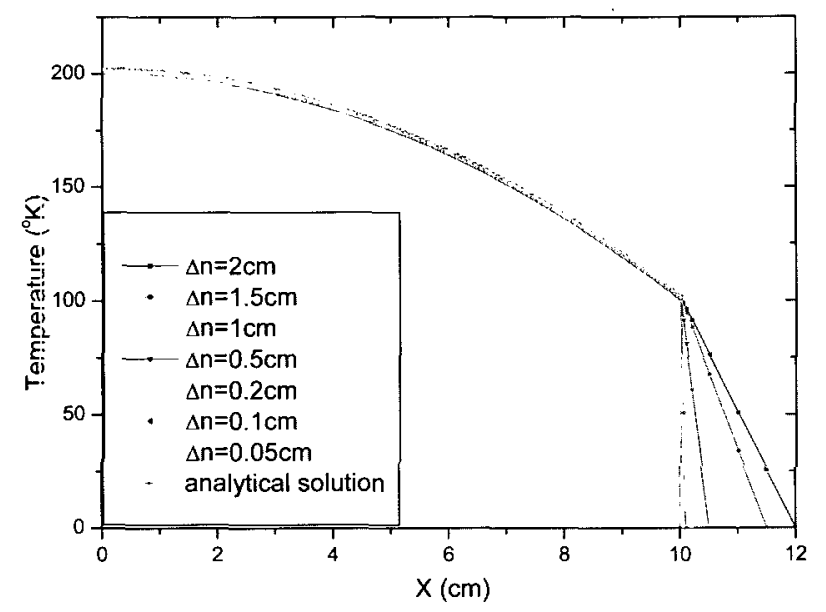

Fig. 5. Transport Solutions and the Analytical Solution of the Convective Boundary Condition Problem

In Fig. 5, the transport solutions with different added layer thickness values provide nearly the same solution close to the analytical result over heat conduction domain (in the added layer, a linear temperature distribution is assumed). Thus, transformation of the convective boundary condition to the fixed-temperature boundary condition can be done safely, and the thickness of the added layer can be chosen rather insensitively.

\section{NUMERICAL RESULTS}

Many calculations were performed; the results for these are available in [14]. Here, only the calculation results of the heat transfer in a PBMR fuel sphere are shown. The problem description can be found in the Appendix .

A Pebble Bed Modular Reactor (PBMR) is a type of high temperature gas cooled reactor fueled by sphere pebbles and cooled by helium [10]. The calculation of the temperature distribution within a fuel pebble is important in an analysis of the design and safety of these reactors. However, due to the heterogeneity of the TRISO-particle distribution in the fuel sphere, prediction of the temperature distribution will be very difficult. Analytical approaches would involve homogenization of the fuel. One homogenized or effective heat conductivity is the volumetric average value; another is the harmonic average value. They give different temperature distributions [15]. Here, the temperature distribution is calculated "heterogeneously" by the transport approximation method with the results compared to analytical solutions based on homogenization.

For the analytical solution, the volume-averaged heat conductivity of the fuel region is given by

$$
\bar{K}=\frac{\sum V_{i} K_{i}}{\sum V_{i}},
$$

and the harmonic averaged heat conductivity is given by

$$
\bar{K}=\left(\frac{\sum V_{i} K_{i_{i}}^{-1}}{\sum V_{i}}\right)^{-1},
$$

where $V_{i}, K_{i}$ are the volume and heat conductivity of material region $i$, respectively .

With the averaged heat conductivity and the uniform heat source distribution, the temperature distribution can be obtained as a function of the radius:

$$
T=T\left(r_{s}\right)
$$

Assuming $T\left(r_{\mathrm{s}}\right)$ is the surface temperature of the TRISOparticle located at the center, the center temperature of the particle is calculated with this boundary value. This gives the highest temperature over the pebble.

In the transport calculation, first the convective boundary condition is transformed to the fixed-temperature boundary condition by adding a conduction layer with a thickness of $0.1 \mathrm{~cm}$. Second, one TRISO particle is located at the center and the others are randomly distributed within the fuel region. The distribution randomness is given by Fine Lattice Stochastic Modeling (FLS) using a cubic lattice [16] (see Appendix). Third, $\alpha=20$ is set in Eqs. (11), (12), and the boundary correction is skipped (as a first-cut calculation). Fourth, the center temperature of the center particle is deduced from the surface temperature of the center particle (radius of the center particle $r_{\mathrm{s}}=0.039 \mathrm{~cm}$ ).

The transport result is selected as one representative from many configurations. Although in each calculation the TRISO-particle distribution differs due to randomness, the temperature distributions are nearly the same [14]. 
The calculation conditions and the results are shown in Table 1 and Fig. 6 . In Fig. 6, the transport result is between the "volume-averaged heat conductivity" model result and the "harmonic-averaged heat conductivity" result. Therefore, the effective heat conductivity of the fuel region should be between the volumetric average and the harmonic average. It is important to note that the volume-averaged heat conductivity is used in the literature [17], resulting in an underestimation of the temperature distribution as compared to the Monte Carlo calculation results in this study.

Table 1. Calculation Conditions of the Pebble Heat Transfer Problem

\begin{tabular}{l|c}
\hline Code & MCNP5 \\
\hline $\mathrm{CPU}$ & Intel $3.2 \mathrm{GHz}$ \\
\hline Number of particles & $1,000,000$ \\
\hline Computing time & 388.23 minutes \\
\hline Standard deviation range & $0.585 \% \sim 0.753 \%$ \\
\hline
\end{tabular}

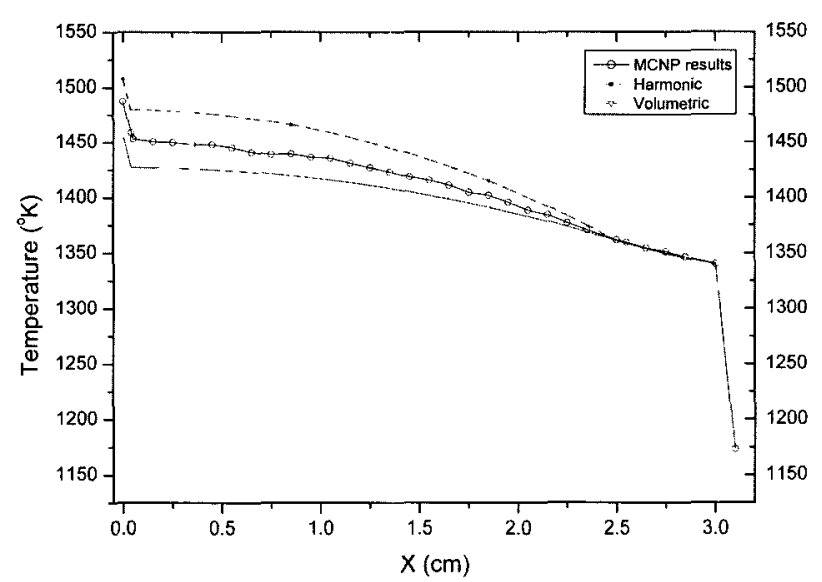

Fig. 6. Two Different Fuel-mixture Model Results and the Transport Approximation Result

\section{CONCLUSIONS}

The Monte Carlo approximation method has been described and shown to be appropriate when tested on a type of heat conduction problem with a prescribed temperature or convective boundary conditions. In particular, the scaling effect was analyzed and a boundary correction was proposed, both of which use the knowledge of the diffusion approximation of transport theory in reactor physics. The method is meshless, and thus can be used with problems with complicated internal geometries easily. For typical problems, other techniques such as FDM and FEM can be used to provide direct and fast results; however, there are problems for which these techniques are not proper or convenient, such as heat transfer within a PBMR fuel sphere, which exhibits very complicated geometry. Due to the capability of handling very complex geometry, the Monte Carlo method proposed in this paper can be applied to these types of problems. To demonstrate this, an example problem with one pebble with many randomly distributed TRISO-particles was solved. For users who are skilled with MCNP, the method may be extended and refined to calculate neutron/heat-coupled problems.

In a future work, an efficient method for temperature profile calculations (at arbitrary positions, e.g., at the center point of the pebble) could be developed based on the adjoint transport problem [18]. In addition, a more robust boundary correction method and "forced absorption" nonanalog simulation would be worthwhile future work to pursue.

\section{APPENDIX: DESCRIPTION OF THE HEAT} TRANSFER PROBLEM WITHIN A FUEL PEBBLE

This Appendix provides a description of the heat transfer problem within a fuel pebble surrounded by helium coolant. The average power per pebble is 250 $\mathrm{MW} / 2.5 / 330000=303.03 \mathrm{~W}$. It is assumed that each TRISO-particle produces equal amount of power.

Table A1. Parameters of PBMR

\begin{tabular}{l|c}
\hline Reactor power & $250 \mathrm{MW}$ \\
\hline Power peaking factor & 2.5 \\
\hline Pebbles & 330,000 \\
\hline Particles per pebble & 15,000 \\
\hline Helium temperature & $1173^{\circ} \mathrm{K}$ \\
\hline
\end{tabular}

Table A2. Geometry and Parameters of a Fuel Pebble

\begin{tabular}{l|c|c|c}
\hline Medium & Radius $(\mathrm{cm})$ & $K(\mathrm{~W} / \mathrm{cm} \cdot K)$ or $h$ & Note \\
\hline Kemel & $0 \sim 0.018$ & 0.0242 & In a TRISO particle \\
\hline Buffer & $0.018 \sim 0.0275$ & 0.013 & In a TRISO particle \\
\hline Inner PyC & $0.0275 \sim 0.0315$ & 0.052 & In a TRISO particle \\
\hline SiC & $0.0315 \sim 0.035$ & 0.178 & In a TRISO particle \\
\hline Outer PyC & $0.035 \sim 0.039$ & 0.052 & In a TRISO particle \\
\hline Fuel region & $0-2.5$ & - & - \\
\hline Graphite matrix & $0 \sim 3$ & 0.47 & $\begin{array}{c}\text { Convection } \\
\text { coefficient; } \\
W / c^{2} \cdot K\end{array}$ \\
\hline Helium & - & 0.1006 & \\
\hline
\end{tabular}



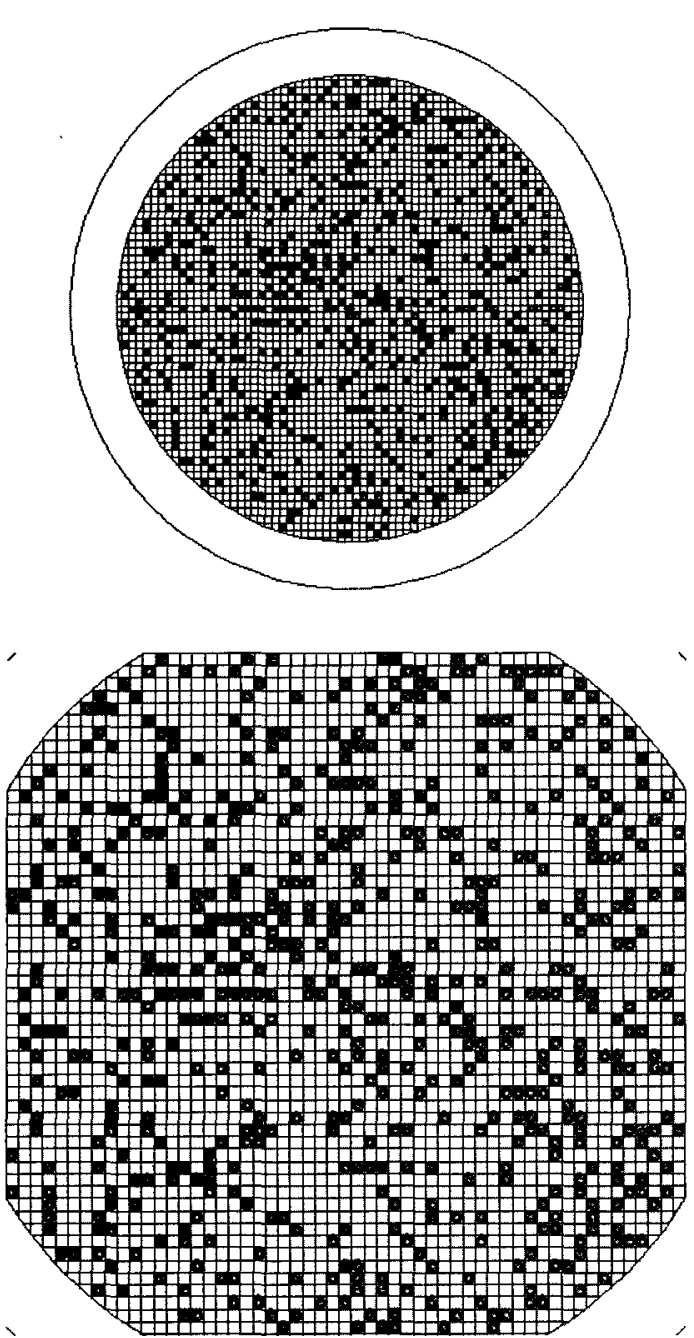

Fig. A1. An X-Y Cross Section View of a Fuel Pebble and Its Particle Distribution On an Enlarged Plane

\section{RIEFERENCES}

[ .1 ] H.S. Carslaw and J.C. Jaeger, Conduction of Heat in Solids, $2^{\text {nd }}$ ed., Oxford, 1959.
[2] James J. Duderstadt and Louis J. Hamilton, Nuclear Reactor Analysis, John Wiley \& Sons, Inc., 1942.

[3] MCNP Manual, LA_UR-03-1987, Los Alamos, 2004.

[4] T.M. Shih, Numerical Heat Transfer, Washington: Hemisphere Pub. Corp., 1984.

[5] S.V. Patankar, Numerical Heat Transfer and Fluid Flow, New York: McGraw-Hill, 1980.

[6] T.J. Hoffman and N.E. Bands, "Monte Carlo Surface Density Solution to the Dirichlet Heat Transfer Problem", Nuclear Science and Engineering, Vol. 59, 1976, pp. 206214.

[7] A. Haji-Sheikh and E.M. Sparrow, "The Solution of Heat Conduction Problems by Probability Methods", ASME Journal of Heat Transfer, Vol. 89, 121, 1967, p.121.

[8] T.J. Hoffman, "Monte Carlo Solution to Heat Conduction Problems with Internal Source", Transactions of the American Society, Vol. 24, 1976, p.181.

[9] S.K. Fraley, T.J. Hoffman, and P.N. Stevens, "A Monte Carlo Method of Solving Heat Conduction Problems", Journal of Heat Transfer, Vol. 102, 1980.

[10] "Current Status and Future Development of Modular High Temperature Gas Cooled Reactor Technology", IAEATECDOC-1198, IAEA, Feb. 2001.

[11] N.Z. Cho, H. Yu, and S. Yun, Nuclear/Thermal Performance Analysis of Pebble Fuel Design for Very High Temperature Gas-Cooled Reactors (VHTGRs), Basic Research Report, KAIST, December 2006.

[12] M. Rama Mohana Rao, Ordinary Differential Equations: Theory and Applications, London: E. Arnold, 1981.

[13] Albert L. Rabenstein, Introduction to Ordinary Differential Equations, $2^{\text {nd }}$ ed. New York, Academic Press, 1972.

[14] J. Shentu, A New Approach to Solve Heat Conduction Problems with Complicated Geometry Based on A Monte Carlo Method, Master Thesis, KAIST, February 2007.

[15] N.Z. Cho and H. Yu, et al., Development of a Neutronics Code Based on Analytic Function Expansion Nodal Method for Pebble-Type High Temperature Gas-Cooled Reactor Design, KAERI/CM-899/2005, KAIST, March 2006.

[16] H. Yu, Fine Lattice Stochastic Modeling of Particle Fuels in HTGR Fuel Element, Master Thesis, KAIST, August 2005.

[17] C.H. Oh, et al, Development of Safety Analysis Codes and Experimental Validation for Very High Temperature GasCooled Reactors, INL/EXT0601362, Idaho National Laboratory, March 2006.

[18] G.I. Bell and S. Glasstone, Nuclear Reactor Theory, Van Nostrand Reinhold, 1970. 